\title{
One Approach to Construction of Bilateral Approximations Methods for Solution of Nonlinear Eigenvalue Problems
}

\author{
Bohdan Mykhajlovych Podlevskyi \\ Institute of Applied Problems of Mechanics \& Mathematics, National Academy of Sciences of Ukraine, Lviv, Ukraine \\ Email: podlev@iapmm.lviv.ua
}

Received December 28, 2011; revised February 8, 2012; accepted February 15, 2012

\begin{abstract}
In this paper a new approach to construction of iterative methods of bilateral approximations of eigenvalue is proposed and investigated. The conditions on initial approximation, which ensure the convergence of iterative processes, are obtained.
\end{abstract}

Keywords: Nonlinear Eigenvalue Problem; Derivatives of Matrix Determinant; Numerical Algorithm of Alternate Approximations

\section{Introduction}

Many theoretical and applied problems of mathematical physics, mechanics and engineering sciences lead to eigenvalue problems. The class of self-adjoint eigenvalue problems, perhaps the most important class of problems because numerous problems that occur in practice belong to this class. However, the eigenvalue problems that are important in practice, very rarely can be solved in a closed form, as a rule one must use numerical methods to solve them. Most numerical methods simply provide approximations to eigenvalues, but they do not make it possible to state how far the calculated eigenvalue is from the true one. Since the self-adjoint eigenvalue problem can have only real eigenvalues, the problem of obtaining approximations and corresponding error estimations of approximation accuracy is equivalent to the determination of upper and lower bounds for the eigenvalues.

Large interest in the eigenvalue bounds, take not only mathematicians, but also physicists, chemists, and engineers and it has many reasons. We name at least two of them here:

1) Lower bounds for eigenvalues are necessary in order to compare predictions of physical theories with experimental results; fine structure corrections in quantum mechanical problems.

2) The knowledge of boundaries for eigenvalues makes it possible in many cases to estimate the reliability of iterative approximation, that is at every step of iterative process to obtain the comfortable a posteriori estimate of error calculations.

For a large class of problems, good upper bounds for positive eigenvalues can be determined relatively easy by means of the Rayleigh-Ritz procedure. To estimate the accuracy of approximations, which are obtained by the Rayleigh-Ritz method, it is important to know at least rough approximations from below. Basically, there are three classes of methods for calculation the lower bounds for eigenvalues (disregarding the methods with a very limited scope of application):

1) The methods based on inclusion theorem.

2) The method of intermediate problems.

3) The Fichera method.

The methods based on inclusion theorems go back to G. Temple [1], L. Collatz [2], and N.J. Lehmann [3,4]. For these methods we need of rough a priori information on the localization of one of the eigenvalues, for example, we need to know: $l_{2}$-the lower bound of $\lambda_{2}$ and $(\psi, A \psi)<l_{2}, \psi \in D(A),\|\psi\|=1$, then

$$
\lambda_{1} \geq(\psi, A \psi)-\frac{\left(\psi, A^{2} \psi\right)-(\psi, A \psi)^{2}}{l_{2}-(\psi, A \psi)} .
$$

In this direction we should note the work of A. V. Knyazev [5,6], B. N. Parlett [7], Behnke H. [8,9], Marmorino M.G. [10].

The method of intermediate problems was proposed by A. Weinstein [11]. Later on the method has been refined by N. Bazly, D. W. Fox, C. Beattie, and others (see [12$14]$ and the bibliography in $[10,14,15])$. For this method one needs the eigenvectors of a neighbouring eigenvalue problem in a closed form. The essence of the method of intermediate problems is as follows: along with the selfadjoint operator $A$ whose eigenvalues are sought, the operator $A^{(0)}$ with known eigenvalues and eigenvectors, 
and such that the operator $A-A^{(0)}$ is positive, is considered. $A^{(0)}$ is called a basic operator. Its range probably lies below the spectrum of the operator $A$. Once the basic operator is selected, by means of the finite perturbation $A_{n}$ a monotonous sequence of intermediate operators $A^{(n)}=A^{(0)}+A_{n}$ spectra of which approximate the spectrum of operator $A$ from below is constructed.

The method, different from the method of intermediate problems was proposed by G. Fichera [16]. This method does not require constructing a base operator with a known spectrum, but has a narrower scope of application. It is applied to the operator $A$, the inverse to which is a compact operator, and the problem $G u-\omega u$, where $G=A^{-1}, \omega=1 / \lambda$, is solved. Then

$$
1 \backslash \lambda_{1}<\cdots<\omega_{1}^{(n)}<\cdots<\omega_{1}^{(1)} .
$$

This paper presents a new approach to construction the methods and algorithms of bilateral approximations of eigenvalues for nonlinear (with respect to spectral parameter) eigenvalue problems, wich have supra-linear speed of convergence. This approach does not use the concepts and apparatus of interval analysis (see, e.g. [20, 21]).

The idea of the approach proposed is that for a continuous monotone in a neighborhood of simple zero $\lambda^{*} \in[a, b]$ function $f:[a, b] \rightarrow R$, which describes the nonlinear equation some auxiliary function $g:[a, b] \rightarrow R$ that has the same zero as the function $f$ and such necessary properties that allow one to build the iterative processes which give monotonous bilateral (alternating or including) approximations to the root of a nonlinear equation [17-19] is constructed and investigated.

Within this approach, the algorithms of bilateral analogues of Newton's method for finding the eigenvalues of nonlinear spectral problems are constructed and justified. The conditions on the initial approximations which provide the alternate approximations to the eigenvalue from two sides and ensure the convergence of iterative process, are obtained.

\section{Statement of the Problem and Some Preliminary Results}

We consider the nonlinear eigenvalue problem

$$
\boldsymbol{D}(\lambda) y=0,
$$

where $\boldsymbol{D}(\lambda)$ is a square matrix of order $n$, all elements of which are sufficiently smooth (at least twice continuously differentiable) functions of the parameter $\lambda \in R, y \in R^{n}$. Eigenvalues sought for solutions of the determinant equation

$$
f(\lambda) \equiv \operatorname{det} \boldsymbol{D}(\lambda)=0 .
$$

To determine the isolated eigenvalue of matrix we propose and justify the Newton-type iterative processes that give the alternate approximations to the root of the Equation (2), i.e.

$$
\begin{aligned}
& \lambda_{0}<\lambda_{2}<\cdots<\lambda_{2 m}<\cdots<\lambda^{*}<\cdots<\lambda_{2 m-1} \\
& <\cdots<\lambda_{3}<\lambda_{1}
\end{aligned}
$$

or

$$
\begin{aligned}
& \lambda_{1}<\lambda_{3}<\cdots<\lambda_{2 m-1}<\cdots<\lambda^{*}<\cdots<\lambda_{2 m} \\
& <\cdots<\lambda_{2}<\lambda_{0}
\end{aligned}
$$

and the including monotonous bilateral approximations to the root, i.e.

$$
\begin{aligned}
& \mu_{0}<\mu_{1}<\cdots<\mu_{2 m}<\cdots<\lambda^{*}<\cdots<v_{2 m} \\
& <\cdots<v_{1}<v_{0}
\end{aligned}
$$

without revealing in so doing the determinant $\operatorname{det} \mathbf{D}(\lambda)$. This means that the left hand side of Equation (2) in explicit form is not set, but the algorithm of finding the functions $f(\lambda)$ and thein derivatives $f^{\prime}(\lambda)$ and $f^{\prime \prime}(\lambda)$ at a fixed value of the parameter $\lambda$, using the $L U$-decomposition of the matrix $\boldsymbol{D}(\lambda)$ is proposed. This algorithm is based on the fact that the matrix $\boldsymbol{D}(\lambda)$ of the order $n$, in which at any given value $\lambda=\lambda_{m}$ the principal minors of all orders from 1 to $(n-1)$ differ from zero, by $L U$-decomposition can be written as

$$
\boldsymbol{D}(\lambda)=\boldsymbol{L}(\lambda) \boldsymbol{U}(\lambda)
$$

where $\boldsymbol{L}(\lambda)$ is the lower triangular matrix with single diagonal elements, and $\boldsymbol{U}(\lambda)$ is the upper triangular matrix. Then

$$
f(\lambda)=\operatorname{det} \boldsymbol{L}(\lambda) \operatorname{det} \boldsymbol{U}(\lambda)=\prod_{i=1}^{n} u_{i i}(\lambda)
$$

Since the elements of a square matrix $\boldsymbol{D}(\lambda)$ (and, therefore, the matrix $\boldsymbol{U}(\lambda))$ are differentiable function, with respect to $\lambda$, then for any $\lambda$ we obtain that

$$
\begin{aligned}
& f^{\prime}(\lambda)=\sum_{k=1}^{n} v_{k k}(\lambda) \prod_{i=1, i \neq k}^{n} u_{i i}(\lambda), \\
& f^{\prime \prime}(\lambda)=\sum_{k=1}^{n} w_{k k}(\lambda) \prod_{i=1, i \neq k}^{n} u_{i i}(\lambda) \\
& +\sum_{k=1}^{n} v_{k k}(\lambda)\left(\sum_{j=1, j \neq k}^{n} v_{j j}(\lambda) \prod_{i=1, i \neq k, i \neq j}^{n} u_{i i}(\lambda)\right)
\end{aligned}
$$

where $v_{i i}(\lambda)=u_{i i}^{\prime}(\lambda)$ and $w_{i i}(\lambda)=v_{i i}^{\prime}(\lambda)$ are the elements of matrices $\boldsymbol{V}(\lambda)$ and $\boldsymbol{W}(\lambda)$ in such decompositions

$$
\begin{aligned}
& D=L U, \\
& B=M U+L V, \\
& C=N U+2 M V+L W,
\end{aligned}
$$

whence we obtain 


$$
\begin{aligned}
f\left(\lambda_{m}\right)= & \prod_{i=1}^{n} u_{i i}, \quad f^{\prime}\left(\lambda_{m}\right)=\sum_{k=1}^{n} v_{k k} \prod_{i=1, i \neq k}^{n} u_{i i}, \\
f^{\prime \prime}\left(\lambda_{m}\right) & =\sum_{k=1}^{n} w_{k k} \prod_{i=1, i \neq k}^{n} u_{i i} \\
& +\sum_{k=1}^{n} v_{k k}\left(\sum_{j=1, j \neq k}^{n} v_{j j} \prod_{i=1, i \neq k, i \neq j}^{n} u_{i i}\right) .
\end{aligned}
$$

The elements of matrices in the decompositions (5) can be calculated using the corresponding recurrent relations written out in [22] (see also [23,24]).

So, not knowing the explicit dependence $f(\lambda)$ on $\lambda$, for any fixed $\lambda$ we can find the value of $f(\lambda)$ and its derivatives. Therefore, for solving (2) we can use the methods that apply the derivatives, in particular, to construct the Newton-type methods, which give the bilateral approximation to the solution. This requires further study of the function $f(\lambda)$, which are realized later in the work.

Further, we demand $f(\lambda)$ to be a three times continuously differentiable function of real variable. By $\lambda^{*}$ we denote an accurate simple root of Equation (2) $\left(f\left(\lambda^{*}\right)\right.$ $=0$ ), in some neighborhood of which such behavior of function $f(\lambda)$ is possible.

1). Function $f(\lambda)$ is convex $\left(f^{\prime \prime}(\lambda)>0\right)$ and its derivative is $f^{\prime}(\lambda)<0$.

$2)$. Function $f(\lambda)$ is concave $\left(f^{\prime \prime}(\lambda)>0\right)$ and its derivative is $f^{\prime}(\lambda)<0$.

$3)$. Function $f(\lambda)$ is convex $\left(f^{\prime \prime}(\lambda)>0\right)$ and its derivative is $f^{\prime}(\lambda)<0$.

4). Function $f(\lambda)$ is concave $\left(f^{\prime \prime}(\lambda)>0\right)$ and its derivative is $f^{\prime}(\lambda)<0$.

\section{Auxiliary Function and Its Properties}

Along with $f(\lambda)$ we consider also a function

$$
z(\lambda)=f(\lambda) / f^{\prime}(\lambda)
$$

which obviously has the same zeros as the function $f(\lambda)$. It is easy to verify that $z(\lambda)$ is twice constinuously differentiable at the point of $\lambda^{*}$ for which the relation

$$
z^{\prime}\left(\lambda^{*}\right)=1, \quad z^{\prime \prime}\left(\lambda^{*}\right)=-f^{\prime \prime}\left(\lambda^{*}\right) / f^{\prime}\left(\lambda^{*}\right)
$$

is satisfied and which has the following properties.

Theorem 3.1. Let $\lambda^{*}$ be a simple real root of Equation (2) in some neighborhood $U$ of which for the function $f(\lambda)$ one of the conditions (A)-(D) is satisfied. Then there is a neighborhood of the root $U_{\varepsilon} \subset U$, in which:

1) when the condition (A) or (D) is satisfied, the function $z(\lambda)=f(\lambda) / f^{\prime}(\lambda)$ is a convex and monotonically increasing function, its derivative $z^{\prime}(\lambda)>0$ and it increases monotonically;
2) when the condition (B) or (C) is satisfied, the function $z(\lambda)=f(\lambda) / f^{\prime}(\lambda)$ is a concave and monotonically increasing function, its derivative $z^{\prime}(\lambda)>0$ and it monotonically decreases.

Proof. Let $f(\lambda)$ be a decreasing and convex with respect to $\lambda$ on $U$ function, that is, $f^{\prime}(\lambda)<0$ and $f^{\prime \prime}(\lambda)>0 \quad$ (the case $\left.(\mathrm{A})\right)$.

Since the function

$$
s(\lambda)=\frac{f(\lambda) f^{\prime \prime}(\lambda)}{\left(f^{\prime}(\lambda)\right)^{2}}
$$

at the point $\lambda=\lambda^{*}$ is equal to zero, then because of continuity of $s(\lambda)$ there is such neighborhood of the root

$$
U_{\varepsilon}\left(\lambda^{*}\right)=\left\{\lambda:\left|\lambda-\lambda^{*}\right|<\varepsilon\right\}
$$

in which

$$
|s(\lambda)|=\left|\frac{f(\lambda) f^{\prime \prime}(\lambda)}{\left(f^{\prime}(\lambda)\right)^{2}}\right| \leq q<1 .
$$

It follows that in the neighborhood $U_{\varepsilon}\left(\lambda^{*}\right)$ the function is $z^{\prime}(\lambda)>0$, since

$$
z^{\prime}(\lambda)=1-\frac{f(\lambda) f^{\prime \prime}(\lambda)}{\left(f^{\prime}(\lambda)\right)^{2}} .
$$

Now from the mean value theorem, applied to differentiable functions $z(\lambda)$ on the interval $[\mu, \lambda] \in U_{\varepsilon}\left(\lambda^{*}\right)$ we obtain

$$
z(\lambda)-z(\mu)=z^{\prime}(\xi)(\lambda-\mu), \quad \xi \in[\mu, \lambda],
$$

whence it follows that the function $z(\lambda)$ is an increasing one.

Consider now the behavior of function $z(\lambda)$ in the neighborhood $U_{\varepsilon}\left(\lambda^{*}\right)$, taking into account its image (7). For any $\lambda<\lambda^{*}$ and $\lambda>\lambda^{*}$ we obtain, respectively, the inequalities

$$
\begin{aligned}
z^{\prime}\left(\lambda^{*}\right)-z(\lambda) & =1-1+\frac{f(\lambda) f^{\prime \prime}(\lambda)}{\left(f^{\prime}(\lambda)\right)^{2}} \\
& =\frac{f(\lambda) f^{\prime \prime}(\lambda)}{\left(f^{\prime}(\lambda)\right)^{2}}>0, \\
z^{\prime}(\lambda)-z\left(\lambda^{*}\right) & =1-\frac{f(\lambda) f^{\prime \prime}(\lambda)}{\left(f^{\prime}(\lambda)\right)^{2}}-1 \\
& =-\frac{f(\lambda) f^{\prime \prime}(\lambda)}{\left(f^{\prime}(\lambda)\right)^{2}}>0,
\end{aligned}
$$

since $f(\lambda)>0$ for $\lambda<\lambda^{*}$ and for $\lambda>\lambda^{*} f(\lambda)<0$. From the inequalities (8) it follows that in the neighborhood $U_{\varepsilon} \equiv U_{\varepsilon}\left(\lambda^{*}\right)$ the derivative $z^{\prime}(\lambda)$ is increas- 
ing, and, consequently, the function $z(\lambda)$ is convex in this neighborhood of the root.

Similar statements about the function $z(\lambda)$ and its derivatives we obtain also for the cases (B), (C) and (D). But unlike the cases (A) and (D), in the cases (B) and (C) the function $z(\lambda)$ is concave. The theorem is proved.

Thus, Theorem 3.1 determines the properties of function $z(\lambda)$, and Figure 1 illustrates its behavior depends on the properties of function $f(\lambda)$ in some neighborhood of the root $\lambda^{*}$.

\section{Bilateral Analogues of Newton Method}

Using the properties of functions $z(\lambda)$, we construct the sequence $\left\{\lambda_{m}\right\}$ which has the property (3).

For the cases (A) and (D) iterative process can be rewritten in the form

$$
\begin{aligned}
& \left\{\begin{array}{l}
\lambda_{2 m+1}=\lambda_{2 m}-\frac{f\left(\lambda_{2 m}\right) f^{\prime}\left(\lambda_{2 m}\right)}{f^{\prime}\left(\lambda_{2 m}\right)^{2}-f\left(\lambda_{2 m}\right) f^{\prime \prime}\left(\lambda_{2 m}\right)}, \\
\lambda_{2 m+2}=\lambda_{2 m+1}-\frac{f\left(\lambda_{2 m+1}\right)}{f^{\prime}\left(\lambda_{2 m+1}\right)},
\end{array}\right. \\
& m=0,1,2, \cdots, \lambda_{0} \in\left(\lambda^{*}-\varepsilon, \lambda^{*}\right),
\end{aligned}
$$

and for the cases (B) and (C) as

$$
\begin{aligned}
& \left\{\lambda_{2 m+1}=\lambda_{2 m}-\frac{f\left(\lambda_{2 m}\right)}{f^{\prime}\left(\lambda_{2 m}\right)}\right. \\
& \left\{\lambda_{2 m+2}=\lambda_{2 m+1}-\frac{f\left(\lambda_{2 m+1}\right) f^{\prime}\left(\lambda_{2 m+1}\right)}{f^{\prime}\left(\lambda_{2 m+1}\right)^{2}-f\left(\lambda_{2 m+1}\right) f^{\prime \prime}\left(\lambda_{2 m+1}\right)},\right. \\
& m=0,1,2, \cdots, \lambda_{0} \in\left(\lambda^{*}-\varepsilon, \lambda^{*}\right) .
\end{aligned}
$$

Justification of bilateral convergence of iterative processes provide the following theorems.

Theorem 4.1. Let $\lambda^{*}$ be a simple real root of Equation (2) and let in some neighborhood of the root

$$
U_{\varepsilon}\left(\lambda^{*}\right)=\left\{\lambda:\left|\lambda-\lambda^{*}\right|<\varepsilon\right\}
$$

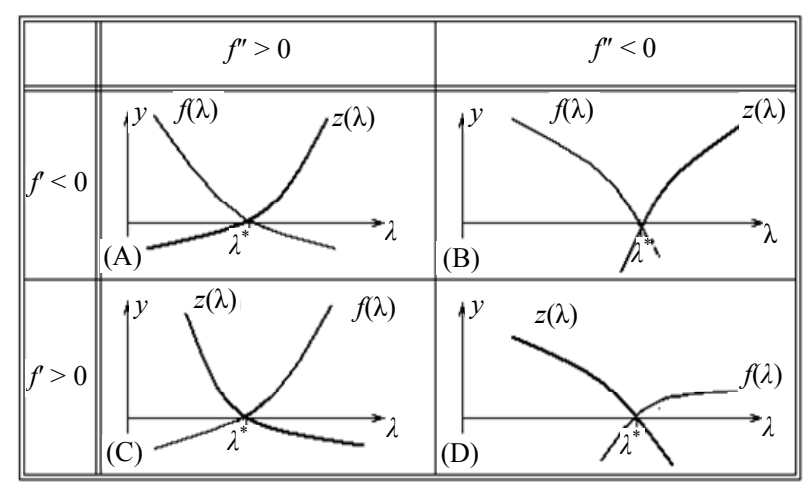

Figure 1. Behavior of the functions $f(\lambda)$ and $z(\lambda)$ in the neighborhood of a simple real root $\lambda^{*}$ of functions $f(\lambda)$. in which

$$
\left|\frac{f(\lambda) f^{\prime \prime}(\lambda)}{f^{\prime}(\lambda)}\right| \leq q<1
$$

for three times continuously differentiable function $f(\lambda)$ that describes the Equation (2.2), the condition (A) or (D) be satisfied, and for the function $z(\lambda)=f(\lambda) / f^{\prime}(\lambda)$ the inequalities

$$
\begin{aligned}
& \frac{z\left(\lambda_{0}\right)}{z^{\prime}\left(\lambda_{0}\right)}>-\frac{2}{M_{2}} \text { for } \lambda_{0} \in\left(\lambda^{*}-\varepsilon, \lambda^{*}\right), \\
& \frac{1}{z^{\prime}\left(\lambda_{1}\right)}>N \text { for } \lambda_{1} \in\left(\lambda^{*}, \lambda^{*}+\varepsilon\right),
\end{aligned}
$$

be satisfied, where

$$
\begin{aligned}
& m_{2}=\min _{\lambda \in \bar{U}_{\varepsilon}\left(\lambda^{*}\right)}\left|z^{\prime}(\lambda)\right|, M_{2}=\max _{\lambda \in \bar{U}_{\varepsilon}\left(\lambda^{*}\right)}\left|z^{\prime \prime}(\lambda)\right|, \\
& N=\max _{\lambda \in\left[\lambda^{*}-\varepsilon, \lambda^{*}\right]}\left|\frac{z(\lambda) z^{\prime \prime}(\lambda)}{z^{\prime}(\lambda)^{2}}\right|<1
\end{aligned}
$$

In addition, let the conditions

$$
\begin{aligned}
& q_{0}=\frac{1}{2}\left(\frac{M_{1} M_{2}^{2}}{m_{1} m_{2}^{2}}\right)^{\frac{1}{3}}\left|\lambda_{0}-\lambda^{*}\right|<1, \\
& q_{1}=\frac{1}{2}\left(\frac{M_{2} M_{1}^{2}}{m_{2} m_{1}^{2}}\right)^{\frac{1}{3}}\left|\lambda_{1}-\lambda^{*}\right|<1
\end{aligned}
$$

be satisfied, where

$$
m_{1}=\min _{\lambda \in \bar{U}_{\varepsilon}\left(\lambda^{*}\right)}\left|f^{\prime}(\lambda)\right|, M_{1}=\max _{\lambda \in \bar{U}_{\varepsilon}\left(\lambda^{*}\right)}\left|f^{\prime \prime}(\lambda)\right| .
$$

Then the iterative process (9), beginning with $\lambda_{0} \in\left(\lambda^{*}-\varepsilon, \lambda^{*}\right)$, convergence to solution $\lambda^{*}$ from both sides

$$
\lambda_{0}<\lambda_{2}<\cdots<\lambda_{2 m}<\cdots<\lambda^{*}<\cdots<\lambda_{2 m-1}<\cdots<\lambda_{3}<\lambda_{1},
$$

and for the errors from the left and from the right the estimations

$$
\left|\lambda_{2 m}-\lambda^{*}\right|<q_{0}^{4^{m}-1}\left|\lambda_{0}-\lambda^{*}\right|,\left|\lambda_{2 m-1}-\lambda^{*}\right|<q_{1}^{4^{m}-1}\left|\lambda_{1}-\lambda^{*}\right| .
$$

are fulfilled, respectively.

Theorem 4.2. Let $\lambda^{*}$ be a simple real root of equation (2) and let in some neighborhood of the root

$$
U_{\varepsilon}\left(\lambda^{*}\right)=\left\{\lambda:\left|\lambda-\lambda^{*}\right|<\varepsilon\right\},
$$

in which

$$
\left|\frac{f(\lambda) f^{\prime \prime}(\lambda)}{f^{\prime}(\lambda)}\right| \leq q<1
$$

for three times continuously differentiable function $(\lambda)$ that describes the Equation (2), the condition (B) or (C) 
to be satisfied, and for the function $z(\lambda)=f(\lambda) / f^{\prime}(\lambda)$ the inequalities

$$
\begin{aligned}
& \frac{1}{z^{\prime}\left(\lambda_{0}\right)}>N_{1} \text { for } \lambda_{0} \in\left(\lambda^{*}-\varepsilon, \lambda^{*}\right), \\
& \frac{z\left(\lambda_{1}\right)}{z^{\prime}\left(\lambda_{1}\right)}<\frac{2}{M_{2}} \text { for } \lambda_{1} \in\left(\lambda^{*}, \lambda^{*}+\varepsilon\right)
\end{aligned}
$$

be satisfied, where

$$
\begin{aligned}
& m_{2}=\min _{\lambda \in \bar{U}_{\varepsilon}\left(\lambda^{*}\right)}\left|z^{\prime}(\lambda)\right|, M_{2}=\max _{\lambda \in \bar{U}_{\varepsilon}\left(\lambda^{*}\right)}\left|z^{\prime \prime}(\lambda)\right|, \\
& N_{1}=\max _{\lambda \in\left[\lambda^{*}, \lambda^{*}+\varepsilon\right]\left|\frac{z(\lambda) z^{\prime \prime}(\lambda)}{z^{\prime}(\lambda)^{2}}\right|<1 .} .
\end{aligned}
$$

In addition, let the conditions

$$
\begin{aligned}
& q_{0}=\frac{1}{2}\left(\frac{M_{2} M_{1}^{2}}{m_{2} m_{1}^{2}}\right)^{\frac{1}{3}}\left|\lambda_{0}-\lambda^{*}\right|<1, \\
& q_{1}=\frac{1}{2}\left(\frac{M_{1} M_{2}^{2}}{m_{1} m_{2}^{2}}\right)^{\frac{1}{3}}\left|\lambda_{1}-\lambda^{*}\right|<1
\end{aligned}
$$

be satisfied, where

$$
m_{1}=\min _{\lambda \in U_{\varepsilon}\left(\lambda^{*}\right)}\left|f^{\prime}(\lambda)\right|, M_{1}=\max _{\lambda \in U_{\varepsilon}\left(\lambda^{*}\right)}\left|f^{\prime \prime}(\lambda)\right|
$$

Then the iterative process (10), beginning with $\lambda_{0} \in\left(\lambda^{*}-\varepsilon, \lambda^{*}\right)$, convergence to the solution $\lambda^{*}$ from both sides

$$
\lambda_{0}<\lambda_{2}<\cdots<\lambda_{2 m}<\cdots<\lambda^{*}<\cdots<\lambda_{2 m-1}<\cdots<\lambda_{3}<\lambda_{1},
$$

and for the errors from the left hand and from the right hand the estimation

$$
\left|\lambda_{2 m}-\lambda^{*}\right|<q_{0}^{4^{m}-1}\left|\lambda_{0}-\lambda^{*}\right|,\left|\lambda_{2 m-1}-\lambda^{*}\right|<q_{1}^{4^{m}-1}\left|\lambda_{1}-\lambda^{*}\right| .
$$

are fulfilled, respectively.

Remark. Two different iterative processes (9) and (10) were used above to justify the alternate approximation in the ideal case, when the behavior of functions $f(\lambda)$ is known or easily investigates. Then alternate approximations come from $n=0$.

In practice, you can use any one of them for all cases (A)-(D) and regardless of which side (left or right from the root $\lambda^{*}$ ) the initial approximation $\lambda_{0}$ is and the algorithm adapts itself to bilateral approximations but then alternate approximations appear at least with $n=1$.

\section{Algorithm of Alternate Approximations and Numerical Results}

To test the proposed iterative processes consider the model eigenvalue problem with quadratic dependence on the parameter

$$
D(\lambda)=\lambda^{2} A_{0}+\lambda A_{1}+A_{2}
$$

where

$$
\begin{gathered}
A_{0}=\left(\begin{array}{cccc}
1.00 & 0.17 & -0.25 & 0.54 \\
0.47 & 1.00 & 0.67 & -0.32 \\
-0.11 & 0.35 & 1.00 & -0.74 \\
0.55 & 0.43 & 0.36 & 1.00
\end{array}\right), \\
A_{1}=\left(\begin{array}{llll}
0.22 & 0.02 & 0.12 & 0.14 \\
0.02 & 0.14 & 0.04 & -0.06 \\
0.12 & 0.04 & 0.28 & 0.08 \\
0.14 & -0.06 & 0.08 & 0.26
\end{array}\right), \\
A_{2}=\left(\begin{array}{lllll}
-3.04759 & -2.18791 & -1.94490 & -2.82430 \\
-2.65007 & -2.47248 & -2.35152 & -2.10538 \\
-0.74566 & -0.64236 & -1.31178 & -0.18524 \\
-4.05001 & -3.06319 & -2.81219 & -3.77944
\end{array}\right) .
\end{gathered}
$$

The eigenvalues will be found as solutions of the determinant equation

$$
f(\lambda) \equiv \operatorname{det} \boldsymbol{D}(\lambda)=0 .
$$

To this end we use the iterative process of alternate approximations (9), which is represented in equivalent form by replacing the function value and its derivatives at the required points by relations (6), which are obtained as a result of $L U$-decomposition of the matrix $\boldsymbol{D}(\lambda)$ (5). As a result, the iterative process (9) takes the form

$$
\begin{aligned}
& \left\{\begin{array}{l}
\lambda_{2 m+1}=\lambda_{2 m}-\left(\sum_{k=1}^{n} \frac{v_{k k}}{u_{k k}}\right) / \sum_{k=1}^{n}\left(\left(\frac{v_{k k}}{u_{k k}}\right)^{2}-\frac{w_{k k}}{u_{k k}}\right), \\
\lambda_{2 m+2}=\lambda_{2 m+1}-1 / \sum_{k=1}^{n} \frac{\bar{v}_{k k}}{\bar{u}_{k k}},
\end{array}\right. \\
& m=0,1,2, \cdots,
\end{aligned}
$$

where $u_{k k}, v_{k k}, w_{k k}$ are the elements of matrix $\boldsymbol{U}, \boldsymbol{V}$ and $\boldsymbol{W}$ in the decompositions (5) at the fixed $\lambda=\lambda_{2 m}$, and $\bar{u}_{k k}, \bar{v}_{k k}$ are the elements of matrix $\boldsymbol{U}, \boldsymbol{V}$ in this decompositions (5) at the fixed $\lambda=\lambda_{2 m+1}$.

Thus, the algorithm can be written in the following.

\section{Algorithm.}

Step 1. Set the initial approximation $\lambda_{0}$ to the $s$-th eigenvalue of the problem (2.15).

Step 2. for $m=0,1,2, \cdots$ to achieve the accuracy do

Step 3. if $m$ is even.

Step 4. Compute the values $u_{k k}, v_{k k}, w_{k k}$ from the decompositions (5) at $\lambda=\lambda_{2 m}$.

Step 5. Compute the approximation to the eigenvalue $\lambda_{2 m+1}$ by (12).

Step 6. go to Step 10.

Step 7. if $m$ is odd.

Step 8. Compute the values $\bar{u}_{k k}, \bar{v}_{k k}$ from the decom- 
positions (5) at $\lambda=\lambda_{2 m+1}$.

Step 9. Compute the approximation to the eigenvalue $\lambda_{2 m+2}$ by (12).

Step 10. end for $m$.

For numerous initial approximations all the eigenvalues of problem (11) were calculated. They fully coincide with the eigenvalues obtained by the usual Newton's method. But the advantage of the algorithm is, in particular, that they give the bilateral approximations. It can be seen, having considered the Table 1 which shows the results of calculations for four eigenvalues. The first column shows the number of eigenvalue, the second shows the number of iterations, the third and fifth the obtained approximations are indicated, respectively, of the left hand and of the right hand, to the $s$-th eigenvalue at each iteration, including the initial approximation. The value of the $s$-th eigenvalue is given in the fourth column. Calculations were carried out to within $\varepsilon=10^{-6}$.

The table shows that for the 1st and 2 nd eigenvalues for the iterative process we obtain bilateral alternating approximations of the form

$$
\lambda_{1}<\lambda_{3}<\lambda_{5}<\lambda_{7} \leq \lambda^{*} \leq \lambda_{6}<\lambda_{4}<\lambda_{2}<\lambda_{0},
$$

and for the 7 th eigenvalue of the form

$$
\lambda_{0}<\lambda_{2}<\lambda_{4}<\lambda_{6} \leq \lambda^{*} \leq \lambda_{7}<\lambda_{5}<\lambda_{3}<\lambda_{1},
$$

beginning with $m=0$. For the 8 th eigenvalue we have obtained the also bilateral approximations similar as for the 7 th one, but the alternating approximations come from $m=1$, i.e.

$$
\lambda_{2}<\lambda_{4} \leq \lambda^{*} \leq \lambda_{5}<\lambda_{3}<\lambda_{1}<\lambda_{0} .
$$

\section{Conclusions}

Approbation of constructed algorithms on model problems shows their reliability and efficiency, and also advantages in comparison with the usual Newton's method in the sense that at every step of iterative process we obtain two-sided estimates of the desired solution, and hence at each step we obtain comfortable a posteriori error estimates .

The proposed approach can be applied to the linear eigenvalue problems with respect to the spectral parameter, moreover if it is compared with existing approaches for obtaining lower bounds of eigenvalues of self-adjoint spectral problems, the approach in contrast to:

- The methods based on inclusion theorems (Temple, Krylov-Bogolyubov), does not require knowledge of the lower border of the following eigenvalue (assuming that the eigenvalues are arranged in ascending order),

- The method of intermediate problems and its various modifications does not require construction of a basic operator with known eigenvalues so that the differ-

\begin{tabular}{|c|c|c|c|}
\hline \multirow{2}{*}{$s$} & \multirow{2}{*}{$m$} & \multicolumn{2}{|l|}{$\lambda^{*}=2.322749$} \\
\hline & & $\lambda_{2 m+1}$ & $\lambda_{2 m}$ \\
\hline \multirow{4}{*}{1} & 0 & $\left(\lambda_{1}=\right) 2.247743$ & $2.5\left(=\lambda_{0}\right)$ \\
\hline & 1 & $\left(\lambda_{3}=\right) \quad 2.321394$ & $2.344590 \quad\left(=\lambda_{2}\right)$ \\
\hline & 2 & $\left(\lambda_{5}=\right) \quad 2.322749$ & $2.322754 \quad\left(=\lambda_{4}\right)$ \\
\hline & 3 & & $2.322749 \quad\left(=\lambda_{6}\right)$ \\
\hline \multirow{2}{*}{$s$} & \multirow{2}{*}{$m$} & $\lambda^{*}=0.796707$ & \\
\hline & & $\lambda_{2 m+1}$ & $\lambda_{2 m}$ \\
\hline \multirow{4}{*}{2} & 0 & $\left(\lambda_{1}=\right) \quad 0.753785$ & $0.9\left(=\lambda_{0}\right)$ \\
\hline & 1 & $\left(\lambda_{3}=\right) \quad 0.782327$ & $0.844609 \quad\left(=\lambda_{2}\right)$ \\
\hline & 2 & $\left(\lambda_{5}=\right) \quad 0.796646$ & $0.799239 \quad\left(=\lambda_{4}\right)$ \\
\hline & 3 & $\left(\lambda_{7}=\right) \quad 0.796707$ & $0.796707 \quad\left(=\lambda_{6}\right)$ \\
\hline \multirow{2}{*}{$s$} & \multirow{2}{*}{$m$} & $\lambda^{*}=-2.635389$ & \\
\hline & & $\lambda_{2 m}$ & $\lambda_{2 m+1}$ \\
\hline \multirow[t]{3}{*}{8} & 0 & $\left(\lambda_{0}=\right)-2.5$ & $-2.577248 \quad\left(=\lambda_{1}\right)$ \\
\hline & 1 & $\left(\lambda_{2}=\right)-2.647351$ & $-2.634985 \quad\left(=\lambda_{3}\right)$ \\
\hline & 3 & $\left(\lambda_{4}=\right)-2.635390$ & $-2.635389 \quad\left(=\lambda_{5}\right)$ \\
\hline \multirow{2}{*}{$s$} & \multirow{2}{*}{$m$} & $\lambda^{*}=-1.223471$ & \\
\hline & & $\lambda_{2 m}$ & $\lambda_{2 m+1}$ \\
\hline \multirow{4}{*}{7} & 0 & $\left(\lambda_{0}=\right)-1.4$ & $-1.150079 \quad\left(=\lambda_{1}\right)$ \\
\hline & 1 & $\left(\lambda_{2}=\right)-1.279845$ & $-1.210666\left(=\lambda_{3}\right)$ \\
\hline & 2 & $\left(\lambda_{4}=\right)-1.224385$ & $-1.223467 \quad\left(=\lambda_{s}\right)$ \\
\hline & 3 & $\left(\lambda_{6}=\right)-1.223471$ & $-1.223471 \quad\left(=\lambda_{\eta}\right)$ \\
\hline
\end{tabular}

Table 1. Bilateral approximations of the eigenvalues of the problem (11).

ence between self-adjoint operator of the original problem and the base one was a positive operator, and constraction of finite-dimentional perturbation of basic operator,

- Fikera method and its modifications, does not require that the inverse to the original operator was compact operator and its construction.

\section{REFERENCES}

[1] G. Temple, "The Theory of Rayleigh's Principle as Applied to Continuous Systems," Proceedings of the Royal Society A, Vol. 119, No. 782, 1928, pp. 276-293. doi:10.1098/rspa.1928.0098

[2] L. Collatz, "Eigenwertaufgaben mit Technischen Anwendungen," Akademische Verlagsgesellschaft Geest \& Portig K.-G., Leipzig, 1963.

[3] N. J. Lehmann, "Beitrage zur Losung linearer Eigenwertpromleme I," Zeitschrift für Angewandte Mathematik und 
Mechanik, Vol. 29, 1949, pp. 341-356.

[4] N. J. Lehmann, "Beitrage zur Losung Linearer Eigenwertpromleme II," Zeitschrift für Angewandte Mathematik und Mechanik, Vol. 30, No. 1-2, 1950, pp. 1-16. doi:10.1002/zamm.19500300101

[5] A. V. Knyazev, "A Block Algorithm for the Kato-Temple Estimates for the Eigenvalues: Rationale and Application," In: V. V. Voevodin, Ed., Adjoint Equations and Perturbation Theory in Mathematical Physics, in Russian, DCM AS USSR, Moscow, 1985, pp. 127-135.

[6] A. V. Knyazev, V. I. Lebedev and A. L. Skorokhodov, "Temple-Lehmann Methods in Iterative Algorithms," in Russian, DCM AS USSR, Moscow, 1985.

[7] B. N. Parlett, "The Symmetric Eignvalue Problem," Prentice Hall, Englewood Clifs, 1980.

[8] H. Behnke and F. Goerisch, "Inclusions for Eigenvalues of Selfadjoint Problems," In: J. Herzberger, Ed., Topics in Validated Computations, Elsevier, Amsterdam, 1994, pp. 277-322.

[9] H. Behnke, "The Calculation of Guarateed Bounds for Eigenvalues Using Complementary Variational Principles," Computing, Vol. 47, No. 1, 1991, pp. 11-27. doi:10.1007/BF02242019

[10] M. G. Marmorino and R. W. Bauernfeind, "Approximate Lower Bound of the Weinstein and Temple Variety," International Journal of Quantum Chemistry, Vol. 107, No. 6, 2007, pp. 1405-1414. doi:10.1002/qua.21268

[11] A. Weinstein and W. Stenger, "Methods of Intermediate Problems for Eigenvalues. Theory and Ramifications," Academic Press, New York, 1972.

[12] N. W. Bazley and D. W. Fox, "Truncations in the Method of Intermediate Problems for Lower Bounds to Eigenvalues," Journal of Research of the National Bureau of Standards Section B, Vol. 65, No. 2, 1961, pp. 105-111.

[13] C. A. Beattie, “An Extension of Aronszajn's Rule; Slicing the Spectrum for Intermediate Problems," SIAM Journal on Numerical Analysis, Vol. 24, No. 4, 1987, pp. 828-843. doi: $10.1137 / 0724053$
[14] C. A. Beattie and W. M. Greenlee, "Convergence Theorems for Intermediate Problems. II," Proceedings of the Royal Society of Edinburgh: Section A, Vol. 132, No. 5, 2002, pp. 1057-1072.

[15] S. H. Gould, "Variational Methods for Eigenvalue Problems," Oxford University Press, London, 1966.

[16] G. Fichera, "Numerical and Quantitative Analysis," Pitnam Press, London, 1978.

[17] B. M. Podlevskyi, "On One Approach to Building Bilateral Iterative Methods for Solving Nonlinear Equations," in Ukraine, Report NAS of Ukraine, No. 5, 1998, pp. $37-41$.

[18] B. M. Podlevskyi, "About One Way to Build Bilateral Iterative Methods for Solving Nonlinear Equations," in Ukraine, Physicomechanical Fields, Vol. 42, No. 2, 1999, pp. 17-25.

[19] B. M. Podlevskyi, "One Approach to the Construction of the Bilateral Approximations Methods for the Solution of Nonlinear Equations," In: G. S. Ladde, N. G. Madhin and M. Sambandham, Eds., Proceeding of Dynamic Systems \& Applications $I V$, Dynamic Publishers Inc., Atlanta, 2004, pp. 542-547.

[20] G. Alefeld and J. Herzberger, "Introduction to Interval Computations," Academic Press, New York, 1983.

[21] A. Neumaier, "Interval Methods for Systems of Equations," Cambridge University Press, Cambridge, 1990.

[22] B. M. Podlevskyi, "Numerical Methods and Algorithms for Solving Generalized Spectral Problems," Ph.D. Thesis, Institute of Mathematics of NASU, Kyiv, 2011.

[23] B. M. Podlevskyi, "On Certain Two-Sided Analogues of Newton's Method for Solving Nonlinear Eigenvalue Problems," Computational Mathematics and Mathematical Physics, Vol. 47, No. 11, 2007, pp. 1745-1755. doi:10.1134/S0965542507110024

[24] Z. Bohte, "Numerical Solution of Some Two-Parameter Eigenvalue Problems," In: P. Gosar and L. Suklje, Eds., Spominski Zbornik Antona Kuhlja, Slovenian Academy of Science and Art, Ljubljana, 1982, pp. 17-28. 